\title{
Joint Optimization of Robot Design and Motion Parameters using the Implicit Function Theorem
}

\author{
Sehoon $\mathrm{Ha}^{*}$, Stelian $\operatorname{Coros}^{\dagger}$, Alexander Alspach*, Joohyung Kim* and Katsu Yamane* \\ *Disney Research, USA \\ Email: \{sehoon.ha,alex.alspach,joohyung.kim,kyamane\}@ disneyresearch.com \\ ${ }^{\dagger}$ Robotics Institute at Carnegie Mellon University, USA \\ Email: scoros@andrew.cmu.edu
}

\begin{abstract}
We present a novel computational approach to optimizing the morphological design of robots. Our framework takes as input a parameterized robot design and a motion plan consisting of trajectories for end-effectors, as well as optionally, for its body. The algorithm we propose is used to optimize design parameters, namely link lengths and the placement of actuators, while concurrently adjusting motion parameters such as joint trajectories, actuator inputs, and contact forces. Our key insight is that the complex relationship between design and motion parameters can be established via sensitivity analysis if the robot's movements are modeled as spatio-temporal solutions to optimal control problems. This relationship between form and function allows us to automatically optimize robot designs based on specifications expressed as a function of range of motion or actuator forces. We evaluate our model by computationally optimizing two simulated robots that employ linear actuators: a manipulator and a large quadruped. We further validate our framework by optimizing the design of a small quadrupedal robot and testing its performance using a hardware implementation.
\end{abstract}

\section{INTRODUCTION}

In the not-so-distant future, a rich ecosystem of robots for service, search and rescue, personal assistance and education have the potential to improve many aspects of our lives. The process of creating new types of robots, however, is notoriously challenging because their motor capabilities are intimately related to how they are designed. For this reason, creating new robots requires a great deal of experience, and is a largely manual and time-consuming task. This tedious and error-prone approach to creating robots is unfortunately necessitated by the lack of formal models that can predict the complex interactions between the design of a robot and its ability to effectively serve its intended purpose.

Rather than relying on trial-and-error approaches, we seek to develop a computational model with the predictive power required to inform design decisions. To achieve this goal, we must establish a relationship between the form and function of robotic devices. To this end, we model a robot's movements as spatio-temporal solutions to optimal control problems. The sensitivities of these optimal solutions enable a guided exploration of the manifold that relates a robot's morphological design parameters and its motor capabilities (Fig. 1).

To test the effectiveness of our computational approach, we use it to optimize the design of linearly actuated robotic devices, a manipulator and a large quadruped, with the goal of minimizing the actuator forces required to drive desired

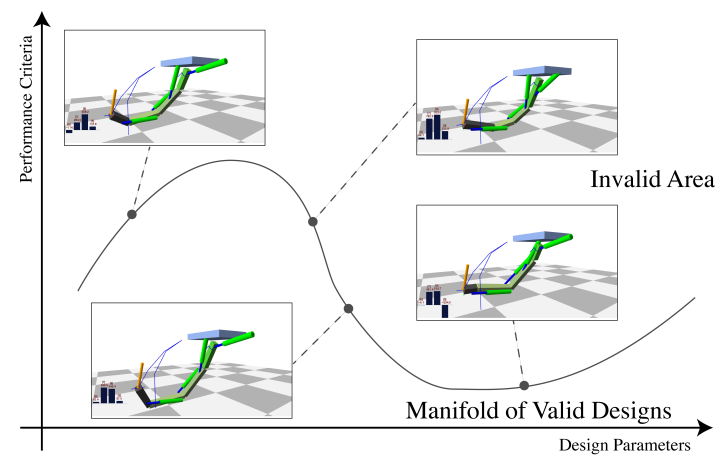

Fig. 1. A set of motion and task constraints implicitly define a manifold of valid designs.

motions. Further, to validate the result of our simulations, we create an optimized design for a small quadrupedal robot, and compare the performances of the original and optimized designs on fabricated hardware.

\section{RELATED WORK}

Designing hardware and motion of robots is a complex task which needs to consider numerous parameters and nonintuitive relationships between them. To obtain a good initial guess for this challenging problem, robot designers often get inspiration from creatures in nature. Under this paradigm, many robots have been successfully designed and built by mimicking morphology and locomotion of real-life animals including salamanders [8], cheetahs [26], kangaroos [11], and chimpanzees [18]. Although these animals can be good sources of inspiration, designing robots is still a time-consuming process that requires repetitive design revisions and performance tests. In this work, we focus on providing an intuitive design framework that allows editing with shorter cycles by identifying relationships between design parameters and performance criteria.

To overcome the complex problem of designing robots, there exists prior work to automate the design by optimizing morphology for a given performance criterion, such as locomotion speed or energy consumption. In particular, evolutionary algorithms (EA), such as Genetic Algorithm (GA) or Simulated Annealing (SA), have received considerable attention for solving design optimization due to its capability 
to handle discrete changes. This approach has been successfully deployed to find the optimal morphology for various types of robots, including virtual creatures [27], manipulators [19], tensegrity robots [20], and soft robots [6]. Although evolutionary algorithms have been proven to be simple yet effective for exploring various designs, the key limitations are limited guarantee on optimality of the final design and difficulty to reproduce the same results on subsequent trials. Instead of relying on stochastic operations, our framework directly identifies the required changes for optimizing the performance of the robot.

In the robotics community, a number of researchers take the approach of automating the design process by optimizing the morphology of a robot for a given task. In general, this approach solves a large optimization problem that minimizes a performance criterion while satisfying kinematic and dynamic constraints. The task-based design optimization has been widely applied to manipulators [22, 5, 28] and parallel manipulators [17, 7, 30] for reaching desired workspace and avoiding joint singularities. However, there exist only a few studies on under-actuated robots, such as pipe-cleaning robots [15], stair-climbing mobile robots [16], virtual creatures [29, 10], or quadrupeds [12] due to complexity of required models.

Such monolithic design optimization is often not easy to use in practice. One of the main problems is that this approach usually takes long time (a few hours for simple robots) to optimize the design. Furthermore, as the first run of optimization rarely results in a satisfactory design, the user needs to repeat the optimization many times while adjusting the objective function. Instead, our framework provides an iterative editing method that the user can provide the change directions any time during the optimization process.

Two of the three examples used in this paper utilize linear actuators, motivated by the recent robots empowered by hydraulic or electronic linear actuators to realize large joint torques in manipulators [2], humanoids [1], and quadrupeds [25, 24, 3, 4]. One of the difficulties of using linear actuators is finding their optimal layout to realize large moment arms while achieving the necessary range of motion, which is not intuitive due to nonlinear relationships between joint positions and forces. The examples demonstrate that our framework can effectively handle linear actuators by including their attachment locations as part of the design parameters.

\section{BACKGROUND: IMPLICIT FUNCTION THEOREM}

The implicit function theorem [13] is a tool for converting an implicitly defined relationship between two sets of variables to an explicit function. Let the following implicit function $\mathbf{f}$ : $\mathcal{R}^{n+m} \rightarrow \mathcal{R}^{m}$ be a relationship between two sets of variables, $\mathbf{y} \in \mathcal{R}^{n}$ and $\mathbf{z} \in \mathcal{R}^{m}$ :

$$
\mathbf{f}(\mathbf{y}, \mathbf{z})=\mathbf{0} \text {. }
$$

For the given implicit relationships $\mathbf{f}$, our goal is to convert it to an explicit function $\mathbf{g}: \Delta \mathbf{y} \mapsto \Delta \mathbf{z}$ within a small disk around the current point, $\left(\mathbf{y}_{0}, \mathbf{z}_{0}\right)$. The Jacobian $D \mathbf{f}$ describes linearized changes around the given point as:

$$
(D \mathbf{f})(\mathbf{y}, \mathbf{z})=\left[\frac{\delta \mathbf{f}}{\delta y_{1}}, \cdots, \frac{\delta \mathbf{f}}{\delta y_{n}} \mid \frac{\delta \mathbf{f}}{\delta z_{1}}, \cdots, \frac{\delta \mathbf{f}}{\delta z_{m}}\right]
$$

where $y_{1}, \cdots, y_{n}$ and $z_{1}, \cdots, z_{m}$ are entries of $\mathbf{y}$ and $\mathbf{z}$. When we change $\mathbf{y}_{0}$ and $z_{0}$ by $\Delta \mathbf{y}$ and $\Delta \mathbf{z}$, the change of the function $\Delta \mathbf{f}$ should be zero to remain on the manifold defined by $\mathrm{f}$ :

$$
\begin{aligned}
\Delta \mathbf{f} & =\mathbf{0}=(D \mathbf{f})\left(\mathbf{y}_{0}, \mathbf{z}_{0}\right)[\Delta \mathbf{y} ; \Delta \mathbf{z}] \\
& =\left[\frac{\delta \mathbf{f}}{\delta y_{1}}, \cdots, \frac{\delta \mathbf{f}}{\delta y_{n}}\right] \Delta \mathbf{y}+\left[\frac{\delta \mathbf{f}}{\delta z_{1}}, \cdots, \frac{\delta \mathbf{f}}{\delta z_{m}}\right] \Delta \mathbf{z} .
\end{aligned}
$$

If there are no additional inequality constraints, the explicit function $g$ can be obtained by simply taking the inverse of the Jacobian matrix with respect to $\mathbf{z}$ :

$$
\Delta \mathbf{z}=-\left[\frac{\delta \mathbf{f}}{\delta z_{1}}, \cdots, \frac{\delta \mathbf{f}}{\delta z_{m}}\right]^{-1}\left[\frac{\delta \mathbf{f}}{\delta y_{1}}, \cdots, \frac{\delta \mathbf{f}}{\delta y_{n}}\right] \Delta \mathbf{y} .
$$

For the general $k$ dimensional function $\mathbf{f}: \mathcal{R}^{n+m} \rightarrow \mathcal{R}^{k}$, we could take the Moonre-Penrose pseudoinverse or employ numerical optimization to obtain $\Delta \mathbf{z}$ that satisfies Eq. (3) as we describe in the next section.

\section{Algorithm}

When a robot executes a motion, the robot and motion can be described by a set of design and motion parameters. Our goal here is to develop a tool that can efficiently navigate through the implicitly defined manifold of valid parameters. In this section, we first describe how the implicit function theorem can be applied to interactive robot design, followed by the details of our problem formulation. We then present the details on how to formulate the relationships between various parameters as a linear system using the implicit function theorem, and how to efficiently solve it using the null space of the linear system.

\section{A. Overview}

A robot design can be described by a set of parameters such as link lengths and actuator attachment points. A robot motion, on the other hand, can be described by joint positions, joint torques, and contact forces at every time frame. These variables collectively form a set of design and motion parameters that defines a robot design and its motion.

The design and motion parameters must satisfy various constraints such as the equation of motion. In some cases, the desired motion may be given as end-effector trajectories that provide additional constraints on the end-effector positions at every frame.

These constraints form the implicitly-defined manifold of valid robot designs and their corresponding motions. We apply the implicit function theorem to derive the relationships among the design and motion parameters. By grouping the parameters in various combinations into $\mathbf{y}$ and $\mathbf{z}$, we can compute how to change a subset of parameters in response to the changes in the other parameters while maintaining the constraints. For example, if we form $\mathbf{y}$ by all the design parameters and include 
all motion parameters in $\mathbf{z}$, we can compute how the motion should be changed according to a design change.

We can even perform certain types of optimization using this technique. Consider choosing the maximum force of a specific actuator as the only element of $\mathbf{y}$, and giving a small negative value as $\Delta \mathbf{y}$. In this case, Eq. (4) gives how to concurrently change the design and motion to reduce the peak force. Alternatively, we can choose to fix the design and change only the motion by adding the design parameters to $\mathbf{y}$ and specifying zero changes for them.

This formulation provides an entirely new approach to the robot design problem: the user can choose the subset of parameters and their change directions, and the algorithm will automatically compute how the other parameters should be changed for the system to stay on the constraint manifold. In contrast to traditional numerical optimization, the user no longer has to formulate the cost function for each set of optimization variables, or indirectly manipulate the optimization result by adjusting the weights in the cost function.

\section{B. Problem Description}

1) Parameters: In our problem, we have two sets of parameters: design parameters and motion parameters. Design parameters, such as link lengths 1 and attachment points of linear actuators $\boldsymbol{\alpha}$, define the robot's morphology that is constant over time. On the other hand, motion parameters describe the state and control signals of the motion at each of the $N$ sample frames. Motion parameters include joint positions $\mathbf{q}_{i}$, actuator forces $\boldsymbol{\tau}_{i}$, and contact forces at the $M$ end-effectors $\mathbf{f}_{i, j}(i=1,2, \ldots, N, j=1,2, \ldots, M)$. We assume that only the end-effectors make contact with the environment.

By collecting all the design and motion parameters, we define the parameter vector $\mathbf{x}$ as $\mathbf{x}=$ $\left[\mathbf{1}^{T}, \boldsymbol{\alpha}^{T}, \mathbf{q}_{1}^{T}, \ldots, \mathbf{q}_{N}^{T}, \boldsymbol{\tau}_{1}^{T}, \ldots \boldsymbol{\tau}_{N}^{T}, \mathbf{f}_{1,1}^{T}, \cdots, \mathbf{f}_{N, M}^{T}\right]^{T}$. We denote the size of $\mathbf{x}$ by $P$.

2) Constraints: A set of design and motion parameters is valid only when it satisfies the task and physics constraints.

The first constraint we consider is that the motion of the robot must satisfy the equation of motion. We therefore define the objective function associated with the equation of motion as

$$
w_{i}^{E O M}(\mathbf{x})=\left|\mathbf{M} \ddot{\mathbf{q}}_{i}+\mathbf{c}-\mathbf{R} \boldsymbol{\tau}_{i}-\sum_{j}^{M} \mathbf{J}_{j}^{T} \mathbf{f}_{i, j}\right|^{2}=0
$$

where $\mathbf{M}$ is the joint-space inertia matrix, $\mathbf{c}$ is the sum of gravitational, centrifugal and Coriolis forces, $\mathbf{R}$ is a moment arm matrix that maps actuator forces to joint torques, and $\mathbf{J}_{j}$ is a Jacobian matrix of the position of the $j$ th end-effector with respect to the joint positions. For a robot with rotary actuators, $\mathbf{R}$ becomes an identity matrix. For floating-base robots such as legged robots, $\mathbf{R}$ contains a zero matrix corresponding to the unactuated floating base.

For brevity of representation, we omit the dependency of matrices: for instance, the moment arm matrix $\mathbf{R}$ depends on $\mathbf{l}, \boldsymbol{\alpha}$, and $\mathbf{q}_{i}$. The velocity $\dot{\mathbf{q}}_{i}$ and acceleration $\ddot{\mathbf{q}}_{i}$ are computed from finite differences of $\mathbf{q}_{i}$.

Many tasks can be described by a set of desired end-effector trajectories and its objective function can be defined as:

$$
w_{i, j}^{E E}(\mathbf{x})=\left|\mathbf{e}_{j}\left(\mathbf{l}, \mathbf{q}_{i}\right)-\overline{\mathbf{e}}_{i, j}\right|^{2}=0
$$

where $\mathbf{e}_{j}(*)$ evaluates the $j$-th end-effector position and $\overline{\mathbf{e}}_{i, j}$ is the desired position of the $j$-th end-effector at the $i$-th frame.

In case of cyclic motions such as locomotion, we place an additional constraint that the joint positions for the first and last frames must be identical:

$$
w^{\text {Loop }}(\mathbf{x})=\left|\mathbf{q}_{1}-\mathbf{q}_{N}\right|^{2}=0
$$

In addition to the equality constraints that represent the implicit relationships, we need to define a set of inequality constraints to limit the range of some of the parameters and enforce contact forces to stay within a friction cone. For brevity, we will omit the details of these straightforward inequality constraints.

For robots with rotary actuators, the lower and upper limits for joint positions can be simply defined as bounds on the elements of $\mathbf{q}_{i}$. If the robot includes linear actuators, their joint positions are limited by the stroke ranges. Therefore, we place the following constraint on the positions of each of the $K$ actuators as

$$
l_{k}^{l o} \leq l_{i, k}(\mathbf{x}) \leq l_{k}^{h i}
$$

where $l_{k}^{l o}$ and $l_{k}^{h i}$ are the lower and upper bounds of the $k$-th linear actuator, and $l_{i, k}(*)$ computes its position at the $i$-th frame.

\section{Formulation of Linear System}

A change in any subset of parameters will likely cause violation of some constraints, and such violation must be corrected by changing the other parameters. We apply the implicit function theorem to obtain the linear relationships between the parameters in order to constrain the parameter changes to stay on the manifold. In the following, we refer to the previously defined $H(=N+N \times M+1)$ constraints as $w_{1}, \ldots, w_{H}$, and their gradients with respect to $\mathbf{x}$ as $\mathbf{C}_{1}, \cdots, \mathbf{C}_{H}$.

Defining $\mathbf{f}=\left(\begin{array}{lll}w_{1} & \ldots & w_{H}\end{array}\right)^{T}$ may seem to be the most natural choice to apply the implicit function theorem. Unfortunately, we cannot directly use the objective functions because their gradients are always zero for all parameters on the manifold due to their quadratic forms. Instead, we use the gradient $\mathbf{C}_{i}$ of the constraints as the implicit relationships, where the Jacobian of gradients (i.e. Hessian of $W_{i}$ ) must be equal to zero.

Because the change of the gradient function $\Delta \mathbf{C}_{i}$ should be zero, we can build a linear system of $\Delta \mathrm{x}$ as:

$$
\Delta \mathbf{C}_{i}=\mathbf{D}_{i} \Delta \mathbf{x}=\mathbf{0},
$$

where $\mathbf{D}_{i}$ is the Jacobian of the gradient $\left[\delta \mathbf{C}_{i} / \delta \mathbf{x}\right]$. We apply finite difference to compute $\mathbf{D}_{i}$ because it is impossible or very difficult to analytically compute them. To improve the 
accuracy, however, we use analytical gradients for computing $\mathbf{C}_{i}$ with respect to some of the parameters as detailed in Section IV-F1

The constraints on linear actuator positions (Eq. (8p) are also converted to a linear system of $\Delta \mathrm{x}$ using the chain rule:

$$
l_{k}^{l o} \leq l_{i, k}^{0}+\mathbf{L}_{i, k} \Delta \mathbf{x} \leq l_{k}^{h i}
$$

where $l_{i, k}^{0}$ is the initial actuator position $l_{i, k}\left(\mathbf{x}_{0}\right)$ and $\mathbf{L}_{k}$ is the Jacobian matrix $\delta l_{i, k} / \delta \mathbf{x}$.

By collecting all equality and inequality constraints, the linear system is formulated as follows:

$$
\begin{aligned}
& \mathbf{A}_{1} \Delta \mathbf{x}=\mathbf{b}_{1} \\
& \mathbf{A}_{2} \Delta \mathbf{x} \geq \mathbf{b}_{2},
\end{aligned}
$$

where

$$
\begin{aligned}
\mathbf{A}_{1} & =\left[\begin{array}{cccc}
\delta \mathbf{C}_{1} / \delta x_{1} & \delta \mathbf{C}_{1} / \delta x_{2} & \ldots & \delta \mathbf{C}_{1} / \delta x_{P} \\
\vdots & \vdots & \ddots & \vdots \\
\delta \mathbf{C}_{H} / \delta x_{1} & \delta \mathbf{C}_{H} / \delta x_{2} & \ldots & \delta \mathbf{C}_{H} / \delta x_{P}
\end{array}\right] \\
\mathbf{b}_{1} & =\left[\begin{array}{lll}
0 & \cdots & 0
\end{array}\right]^{T} \\
\mathbf{A}_{2} & =\left[\begin{array}{c}
\mathbf{L}_{1,1} \\
-\mathbf{L}_{1,1} \\
\vdots \\
-\mathbf{L}_{N, K}
\end{array}\right] \\
\mathbf{b}_{2} & =\left[\begin{array}{llll}
l_{1}^{o}-l_{1,1}^{0} & -\left(l_{1}^{h i}-l_{1,1}^{0}\right) & \ldots & -\left(l_{K}^{h i}-l_{N, K}^{0}\right)
\end{array}\right] .
\end{aligned}
$$

Note that the matrices of the formulated linear system are very sparse, because the motion parameters only affect the constraints on the corresponding frames.

The final component of the system is the user input specifying the desired changes of a subset of parameters $\Delta \overline{\mathbf{y}}$. We denote the remaining unknown parameters by $\Delta \mathbf{z}$, which we shall determine such that the constraints are maintained after $\Delta \overline{\mathbf{y}}$ is applied. Without losing generality, we can divide the equality and inequality constraints of Eq. (11) as

$$
\begin{aligned}
& \mathbf{A}_{1 y} \Delta \overline{\mathbf{y}}+\mathbf{A}_{1 z} \Delta \mathbf{z}=\mathbf{b}_{1} \\
& \mathbf{A}_{2 y} \Delta \overline{\mathbf{y}}+\mathbf{A}_{2 z} \Delta \mathbf{z} \geq \mathbf{b}_{2} .
\end{aligned}
$$

\section{Optimization}

Instead of using the pseudoinverse as in Eq. (4), we compute $\mathbf{z}$ by applying numerical optimization because 1) we also have inequality constraints, and 2) there may exist multiple solutions or there may be no exact solution, depending on the size of $\overline{\mathbf{y}}$. The optimization problem can be derived from Eq. (16) as

$$
\begin{array}{cl}
\min _{\Delta \mathbf{z}} & \Delta \mathbf{z}^{T} \mathbf{S} \Delta \mathbf{z} \\
\text { s.t. } & \mathbf{A}_{1 z} \Delta \mathbf{z}=\mathbf{b}_{1}-\mathbf{A}_{1 y} \Delta \overline{\mathbf{y}} \\
& \mathbf{A}_{2 z} \Delta \mathbf{z} \geq \mathbf{b}_{2}-\mathbf{A}_{2 y} \Delta \overline{\mathbf{y}},
\end{array}
$$

where $\mathbf{S}$ is a positive definite, diagonal weight matrix, where we use 0.01 for actuator forces $\tau, 10.0$ for contact forces $\mathbf{f}$, and 1.0 for others.
Although this is a standard quadratic programming problem, it is numerically difficult to solve due to a large number of constraints. We therefore represent the feasible solution space with respect to the linear constraints using the null space of the coefficient matrix $\mathbf{A}_{1 z}$ :

$$
\Delta \mathbf{z}=\Delta \mathbf{z}_{0}+\mathbf{N u}
$$

where $\Delta \mathbf{z}_{0}=\mathbf{A}_{1 z}^{+}\left(\mathbf{b}_{1}-\mathbf{A}_{1 y} \overline{\mathbf{y}}\right)$ and $\mathbf{N}$ represents the null space of $\mathbf{A}_{1 z}$. Note that both $\mathbf{A}_{1 z}^{+}$and $\mathbf{N}$ can be efficiently computed from one singular value decomposition. Eq. (17) can now be reduced into

$$
\begin{array}{ll}
\min _{\mathbf{u}} & \left(\Delta \mathbf{z}_{0}+\mathbf{N u}\right)^{T} \mathbf{S}\left(\Delta \mathbf{z}_{0}+\mathbf{N u}\right) \\
\text { s.t. } & \mathbf{A}_{2 z}\left(\Delta \mathbf{z}_{0}+\mathbf{N u}\right) \geq \mathbf{b}_{2}-\mathbf{A}_{2 y} \Delta \overline{\mathbf{y}}
\end{array}
$$

which can be solved much more efficiently than the original optimization problem.

\section{E. Summary}

Algorithm 1 summarizes the optimization algorithm described in this section. It takes the initial parameters $\mathbf{x}_{0}$ as input, and finds the optimal parameters $\mathbf{x}^{*}$ that realizes the desired change and also maintain the design and motion on the constraint manifold. In Algorithm 1 $\mathcal{E}$ represents the sum of the objective functions:

$$
\mathcal{E}=\sum_{i}^{H} w_{i}(\mathbf{x}) .
$$

A non-zero $\mathcal{E}$ implies violation of constraints due to numerical errors caused by linear approximation. At each iteration, we attempt to correct this error as described in Section IV-F2. We run the optimization until it reaches the maximum iteration (1000 in our implementation), or the error $\mathcal{E}$ is greater than a threshold $\overline{\mathcal{E}}\left(10^{-6}\right)$.

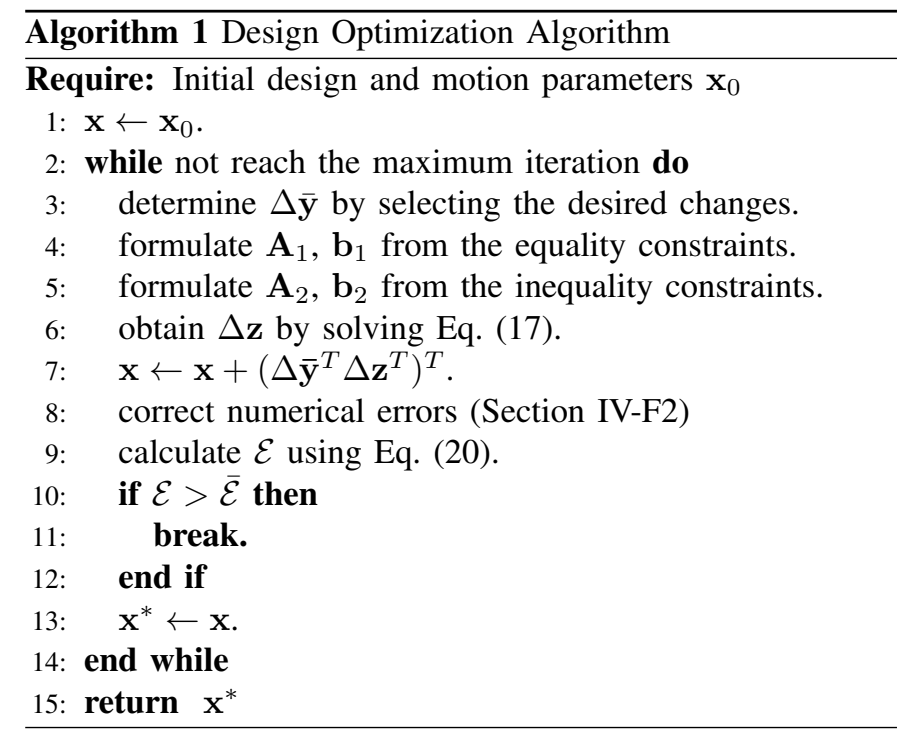

In line 3 of Algorithm [1, we could also let the user interactively choose $\Delta \overline{\mathbf{y}}$ as demonstrated in Section $\mathrm{V}$-A . This version of the algorithm provides an intuitive interface for 
TABLE I

THE PROBLEM PARAMETERS

\begin{tabular}{|c|c|c|c|c|c|c|c|c|c|c|}
\hline \multicolumn{4}{|c|}{ Problem } & \multicolumn{5}{|c|}{ \# Parameters } & \multicolumn{2}{|c|}{ \# Expanded Nodes } \\
\hline Robot & Actuation & \# Actuators & \# Frames & Lengths & $\begin{array}{l}\text { Actuator } \\
\text { Points }\end{array}$ & $\begin{array}{c}\text { Joint } \\
\text { Positions }\end{array}$ & $\begin{array}{l}\text { Actuator } \\
\text { Forces }\end{array}$ & $\begin{array}{c}\text { Contact } \\
\text { Forces }\end{array}$ & $\begin{array}{c}\text { \# Total } \\
\text { Parameters }\end{array}$ & $\begin{array}{l}\text { Time per } \\
\text { Iteration }\end{array}$ \\
\hline Manipulator & Linear & 4 & 7 & 4 & 8 & 28 & 28 & 0 & 68 & $0.8 \mathrm{~s}$ \\
\hline Large Quadruped & Linear & 16 & 13 & 8 & 16 & 208 & 208 & 156 & 596 & $40.2 \mathrm{~s}$ \\
\hline Small Quadruped & Rotary & 14 & 13 & 5 & N/A & 182 & 182 & 120 & 489 & $32.1 \mathrm{~s}$ \\
\hline
\end{tabular}

TABLE II

optimizing the design of a robot by specifying desired changes to any of the design or motion parameters. For example, the user could choose to reduce the maximum torque, or adjust the link length depending on the design requirements.

\section{F. Implementation Note}

1) Computation of Gradients: It is desirable to calculate the analytical gradient vector $\mathbf{C}_{i}$ with respect to all parameters. Unfortunately, most of the constraints are nonlinear and it is difficult to analytically calculate their gradient with respect to some of the parameters, such as the partial derivative of the Coriolis forces with respect to the joint velocities. Instead, we only compute the analytical gradients that can be easily derived. In our experience, this compromise provides enough accuracy while keeping the computational cost reasonable.

For instance, the gradient of end-effector constraints with respect to the link lengths can be simply computed as:

$$
\delta w^{E E} / \delta \mathbf{l}=\mathbf{J}_{j}^{T}\left(\mathbf{e}_{j}(\mathbf{l}, \mathbf{q})-\overline{\mathbf{e}}\right) .
$$

Similarly, the analytical gradients of the equation of motion constraint can be easily calculated for the joint forces and contact forces as:

$$
\begin{aligned}
& \delta w^{E O M} / \delta \boldsymbol{\tau}=\mathbf{R}^{T}\left(\mathbf{M} \ddot{\mathbf{q}}_{i}+\mathbf{c}-\mathbf{R} \boldsymbol{\tau}_{i}-\sum_{j}^{M} \mathbf{J}_{j}^{T} \mathbf{f}_{i, j}\right) \\
& \delta w^{E O M} / \delta \mathbf{f}= \\
& {\left[\mathbf{J}_{1} \cdots \mathbf{J}_{M}\right]^{T}\left(\mathbf{M} \ddot{\mathbf{q}}_{i}+\mathbf{c}-\mathbf{R} \boldsymbol{\tau}_{i}-\sum_{j}^{M} \mathbf{J}_{j}^{T} \mathbf{f}_{i, j}\right) . }
\end{aligned}
$$

2) Correction of Numerical Errors: Because we apply linear approximation to the nonlinear constraints, numerical errors will accumulate and eventually lead to physically invalid solutions. Our framework prevents this numerical deviation by re-optimizing only the motion parameter for the equality constraints.

First, we optimize the joint angles considering only the endeffector constraints (Eq. (6)):

$$
\mathbf{q}_{i}=\underset{\mathbf{q}_{i}}{\operatorname{argmin}} w^{E E} .
$$

We then optimize the actuator forces and contact forces to satisfy the equation of motion (Eq. (5)):

$$
\boldsymbol{\tau}_{i}, \mathbf{f}_{i, 1}, \cdots, \mathbf{f}_{i, M}=\underset{\boldsymbol{\tau}_{i}, \mathbf{f}_{i, 1}, \cdots, \mathbf{f}_{i, M}}{\operatorname{argmin}} w^{E O M} .
$$

Note that the design parameters are not changed in the error correction process.
THE RESULTS ON MANIPULATORS WITH LINEAR ACTUATORS

\begin{tabular}{|c|c|c|c|c|c|c|c|}
\hline & \multicolumn{4}{|c|}{ Link Lengths(cm) } & \multicolumn{2}{c|}{$\begin{array}{c}\text { Actuator } \\
\text { Positions (cm) }\end{array}$} & $\begin{array}{c}\text { Maximum } \\
\text { Force (N) }\end{array}$ \\
\hline Design & Link1 & Link2 & Link3 & Link4 & Link2 & Link3 & Manipulation \\
\hline Initial & 20.0 & 30.0 & 30.0 & 20.0 & 15.0 & 5.0 & $5.89 \times 10^{2}$ \\
\hline Motion Optimized & 20.0 & 30.0 & 30.0 & 20.0 & 15.0 & 5.0 & $4.87 \times 10^{2}$ \\
\hline Design Optimized & 19.7 & 24.4 & 29.5 & 24.9 & 14.5 & 8.3 & $\mathbf{3 . 8 3} \times \mathbf{1 0}^{2}$ \\
\hline Length Edited & $\mathbf{2 4 . 0}$ & 24.4 & 29.1 & 24.9 & 10.8 & 8.3 & $4.80 \times 10^{2}$ \\
\hline
\end{tabular}

\section{EXPERIMENTS}

In this section, we discuss the simulation and hardware experiments for validating the proposed design optimization algorithm. We tested three sets of examples: simulation of linearly actuated manipulators, simulation of linearly actuated quadrupeds, and hardware of quadrupeds with off-the-shelf rotary servos. The algorithm is implemented in Python with the PyDART [23] and SciPy library [14] on Ubuntu Linux, and the computations are conducted on a single core of $3.40 \mathrm{GHz}$ Intel i7 processor. The parameters for all problems are described in Table []

\section{A. Simulation of Manipulators with Linear Actuators}

We first show a proof of concept of our algorithm by optimizing the design of a linearly actuated manipulator. First, we provide an initial manipulator design with link lengths of $20 \mathrm{~cm}, 30 \mathrm{~cm}, 30 \mathrm{~cm}$, and $20 \mathrm{~cm}$. The manipulator has four degrees of freedom fully actuated by four actuators, each of which with $25 \mathrm{~cm}$ body length and $16 \mathrm{~cm}$ stroke. The actuators are monoarticular with $5 \mathrm{~cm}$ moment arms except the second biarticular actuator with a $15 \mathrm{~cm}$ moment arm. The attachment points of actuators can be adjusted vertically, except the points on the base link that can adjusted horizontally. The input task is to move a $5 \mathrm{~kg}$-object along the target trajectory that has the $60 \mathrm{~cm}$ maximum distance from the base link. Note that the initial design is generated based on simple rules rather than pre-tuned for any performance index. In our experience, our framework is not very sensitive to the initial parameters.

In this example, we demonstrate two editing modes: forcedriven mode for reducing the maximum actuator force, and length-driven mode for adjusting the length of the target link.

We begin with the force-driven editing mode, which provides an intuitive interface for editing the design such that the maximum actuator force is reduced. At each iteration, our algorithm selects the maximum force parameter during the entire motion, and set the desired change as $-1 \%$ of its value. Using our algorithm, we are able to find the optimal design that reduces the maximum actuator force from $589 \mathrm{~N}$ to $383 \mathrm{~N}$, which happens at the third actuator. The 

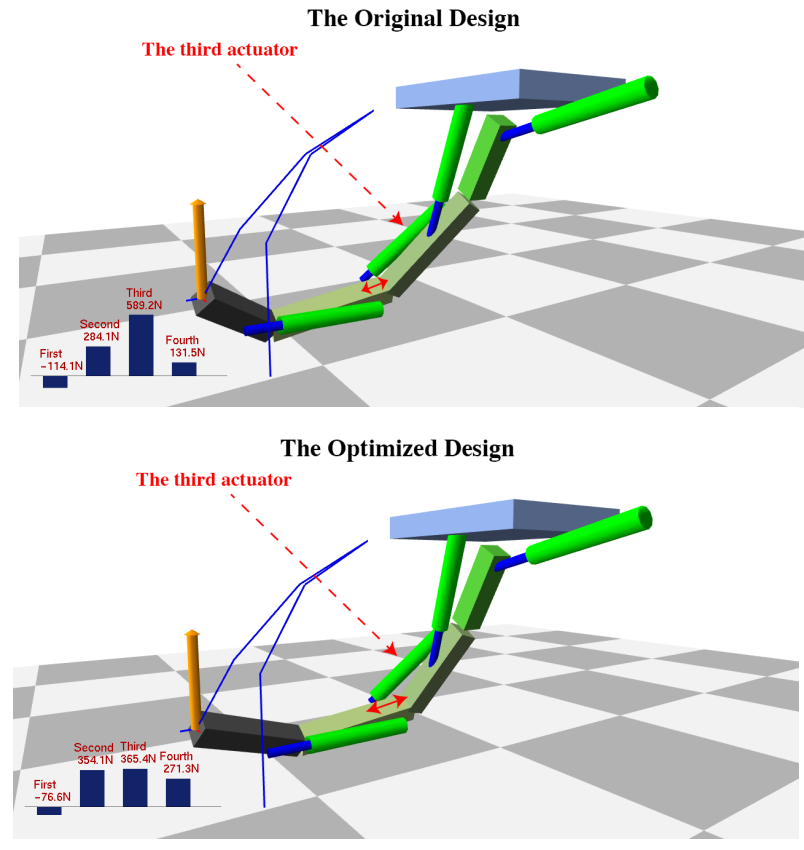

Fig. 2. Comparison of the original (top) and optimized (bottom) manipulators. Note the larger moment arm for the third actuator in the optimized manipulator

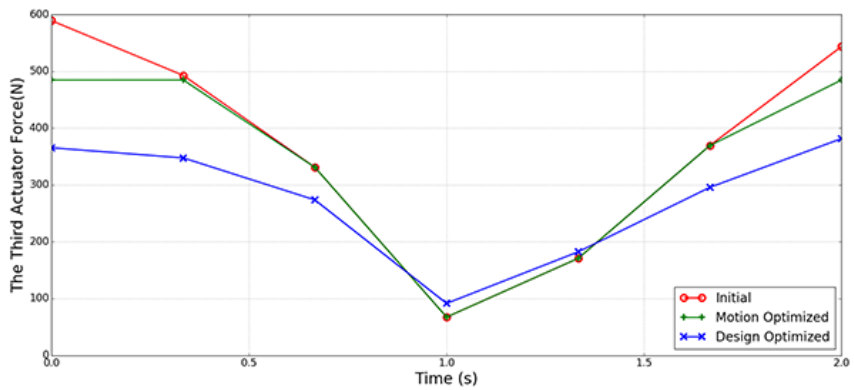

Fig. 3. Force profiles of the third actuator in three designs: the initial design and motion (red), the initial design with optimized motion (green), and the concurrently optimized design and motion (blue). The concurrent optimization of the design and motion parameters results in the most efficient actuator force profile.

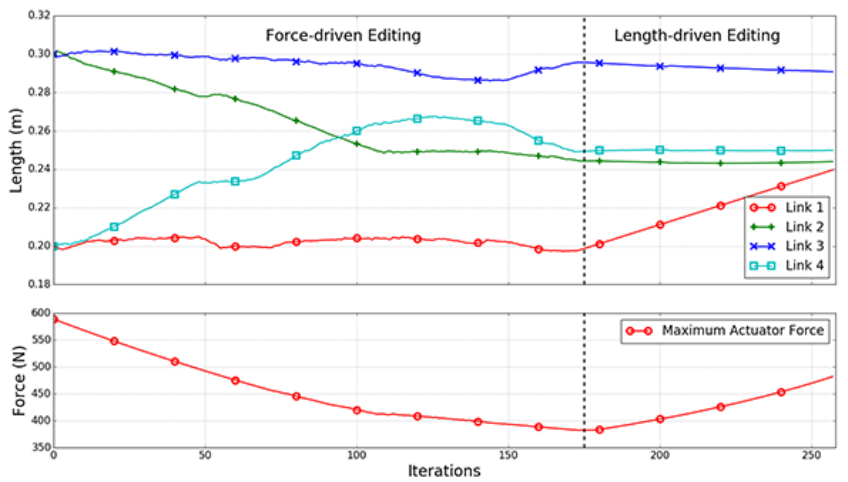

Fig. 4. Changes in link lengths and the maximum actuator force the third actuator during the manipulator design optimization process. The first half was edited in the force-driven mode for minimizing the maximum torque, while the second half was edited in the length-driven mode for increasing the length of the first link. parameters of the resulting design are shown in Table II and the visual comparison is provided in Fig. 2 In general, the algorithm increases the moment arm for the third actuator by changing its attachment point, and adjusts the second and fourth link lengths to secure the necessary range of motion. Further increasing the moment arm of the third actuator cannot maintain the range to perform the entire desired motion, which triggers the break condition in line 10 of Algorithm 1

Our algorithm also allows optimization of the motion parameters only, which gives another pair of design and motion where the design is the same as the initial one but the force profile is different. Figure 3 compares the torque profile of the third actuator for the three pairs of design and motion. Although optimizing only the motion can also reduce the maximum torque, its final result $(487 \mathrm{~N})$ is worse than the result of concurrent design and motion optimization (382 N).

Let us consider the scenario where the user is not satisfied with the proportion of the optimized design and is willing to sacrifice the maximum force to increase the length of the first link. The user can then start the length-driven editing mode and set the desired change of the first link length as $+1 \mathrm{~mm}$. Given the new input, the algorithm successfully increases the link length while properly adjusting other design and motion parameters as shown in the second half of Fig. 4 The maximum achievable length is $24 \mathrm{~cm}$, beyond which the range of motion becomes too small to perform the desired motion. Our algorithm automatically relocates the bottom attachment point of the second actuator closer to the joint in order to obtain the required range of motion.

\section{B. Simulation of Large Quadrupeds with Linear Actuators}

This set of examples involves a large-size quadruped robot with linear actuators. The initial design of the quadruped has $1.2 \mathrm{~m}$ body length and $0.95 \mathrm{~m}$ shoulder height at its rest pose with a mass of approximately $90 \mathrm{~kg}$. Each leg has four degrees of freedom digitigrade configuration with four linear actuators. Two biarticular actuators are attached to the hip joints, and two monoarticular actuators are attached to the knee and ankle joints. All the actuators have the same length $(25 \mathrm{~cm})$ and stroke range $(16 \mathrm{~cm})$ as the actuators for the manipulator example. The design parameters are constrained to be left-right symmetric during the optimization process. We choose a manually-created initial design and generate the input base link and end-effector trajectories by solving space-time optimization using the technique described in Megaro et al. [21]. We treat the motion of the floating base as input.

The goal of this experiment is to optimize the initial design and motion parameters for two different tasks: slow walking with $0.15 \mathrm{~m}$ step length and fast walking with $1.0 \mathrm{~m}$ step length. In both cases, we optimize the design and motion parameters such that the maximum actuator force is reduced.

Our algorithm successfully derives different designs that reduce the maximum torques by $48 \%$ and $40 \%$ for the slow and fast walking tasks respectively (Table III). Figure 5 compares the optimized design and motion while Figure 6 plots the force profile of the knee actuator that requires 
TABLE III

THE RESULTS ON LARGE QUADRUPEDS WITH LINEAR ACTUATORS

\begin{tabular}{|c|c|c|c|c|c|c|c|c|}
\hline & \multicolumn{3}{|c|}{ Link Lengths(cm) } & \multicolumn{2}{c|}{$\begin{array}{c}\text { Actuator } \\
\text { Positions (cm) }\end{array}$} & \multicolumn{2}{c|}{$\begin{array}{c}\text { Maximum } \\
\text { Force (N) }\end{array}$} \\
\hline Design & $\begin{array}{c}\text { Rear } \\
\text { Hip }\end{array}$ & $\begin{array}{c}\text { Rear } \\
\text { Thigh }\end{array}$ & $\begin{array}{c}\text { Rear } \\
\text { Shin }\end{array}$ & $\begin{array}{c}\text { Rear } \\
\text { Foot }\end{array}$ & $\begin{array}{c}\text { Rear } \\
\text { Knee }\end{array}$ & $\begin{array}{c}\text { Rear } \\
\text { Ankle }\end{array}$ & $\begin{array}{c}\text { Slow } \\
\text { Walking }\end{array}$ & $\begin{array}{c}\text { Fast } \\
\text { Walking }\end{array}$ \\
\hline Initial & 15.0 & 30.0 & 30.0 & 20.0 & 4.0 & 4.0 & $1.30 \times 10^{3}$ & $2.15 \times 10^{3}$ \\
\hline Slow Walking & 10.0 & 35.2 & 31.9 & 16.1 & 9.2 & 9.0 & $\mathbf{0 . 6 8} \times \mathbf{1 0}^{3}$ & N/A \\
\hline Fast Walking & 16.1 & 31.2 & 32.2 & 17.1 & 6.1 & 3.3 & $0.89 \times 10^{3}$ & $\mathbf{1 . 2 9}^{3} \times \mathbf{1 0}^{3}$ \\
\hline
\end{tabular}

The Optimized Design for Slow Walking

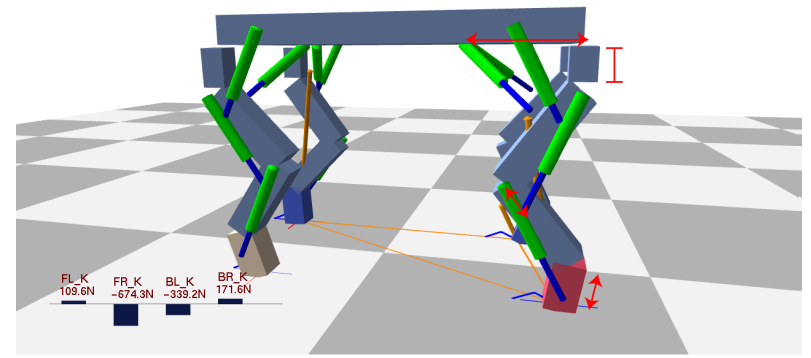

The Optimized Design for Fast Walking

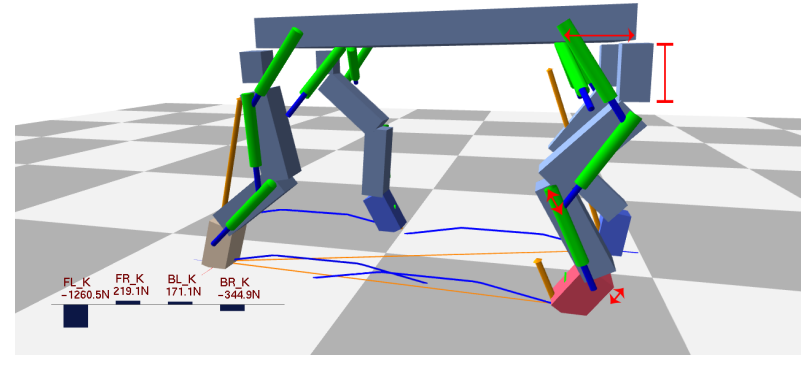

Fig. 5. Comparison of the optimized linearly actuated quadrupeds for slow (top) and fast (bottom) walking tasks. We highlighted a few noticeable differences. In general, the design for slow walking has larger moment arms than the design for fast walking.
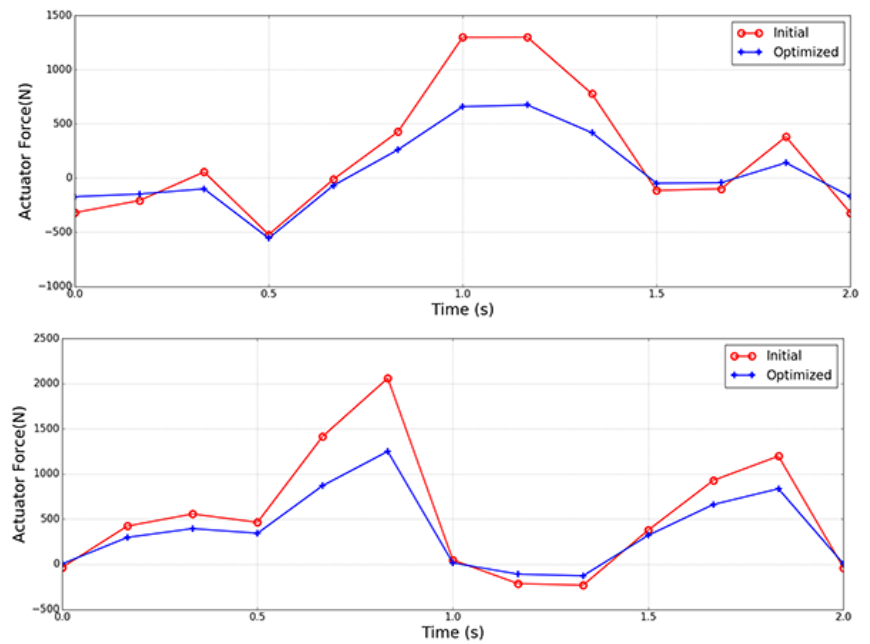

Fig. 6. Force profiles of the back right knee in the slow walking optimized (top) and fast walking optimized (bottom) designs.
TABLE IV

THE RESULTS ON SMALL QUADRUPEDS WITH ROTARY ACTUATORS

\begin{tabular}{|c|c|c|c|c|c|c|c|}
\hline & \multicolumn{4}{|c|}{ Link Lengths(cm) } & \multicolumn{2}{c|}{ Maximum Torque } \\
\hline Design & $\begin{array}{c}\text { Front } \\
\text { Thigh }\end{array}$ & $\begin{array}{c}\text { Front } \\
\text { Shin }\end{array}$ & $\begin{array}{c}\text { Rear } \\
\text { Thigh }\end{array}$ & $\begin{array}{c}\text { Rear } \\
\text { Shin }\end{array}$ & $\begin{array}{c}\text { Rear } \\
\text { Feet }\end{array}$ & $\begin{array}{c}\text { Simulation } \\
\text { Torque (Nm) }\end{array}$ & $\begin{array}{c}\text { Hardware } \\
\text { Current (mA) }\end{array}$ \\
\hline Initial & 8.00 & 16.0 & 8.00 & 8.00 & 8.00 & 2.08 & $(7.66 \pm 0.37) \times 10^{2}$ \\
\hline Optimized & 7.87 & 12.9 & 9.43 & 7.28 & 8.00 & $\mathbf{1 . 4 2}$ & $(5.35 \pm 0.23) \times 10^{2}$ \\
\hline
\end{tabular}

the maximum force in the original design. Please refer to the supplemental video for the details of the metamorphosis process. The most notable difference of the two optimized designs is in their knees: the moment arm is increased in the slow walking quadruped while it is kept small in the fast walking optimized design in order to realize larger range of motion. In addition, the algorithm elongated the rear thigh for the slow walking task, while increasing the length of the rear hip for fast walking. For the slow walking task, we have expected straight-knee configuration similar to those of elephants or rhinos, but the algorithm did not result in such design because the enlargement of moment arm has larger effect than the reduction of the joint torque.

We also cross-validated the optimal designs by reoptimizing the motion parameters with fixed design parameters. As we expected, the fast walking optimized requires $31 \%$ more maximum actuator forces than the slow walking optimized design due to larger moment arms at knees. On the other hand, the slow walking optimized design cannot execute the fast walking task due to its limited range of the motion.

\section{Hardware of Small Quadrupeds with Rotary Actuators}

Finally, we validate our algorithm in hardware by implementing a small-size quadruped with off-the-shelf rotary actuators and 3D printed links. The robot has $20 \mathrm{~cm}$ body length and $24 \mathrm{~cm}$ shoulder height at its rest pose. Each leg has four Dynamixel XM-430 W-210 [9] servos that have $3.7 \mathrm{Nm}$ stall torque at $14.0 \mathrm{~V}$, and each foot is fabricated as rubbercoated hemisphere. For testing purpose, we put four $500 \mathrm{~g}$ weights on the top of the base link. We tether the robot to an external computer and replay the motion plan without any balance controller.

We manually choose an initial design of the quadruped and optimize the design such that the maximum joint torque is minimized. Once again, the initial motion plan is generated using the method described in Megaro et al. [21]. Table IV compares the initial and optimized parameters. Because the initial design requires a maximum torque of $2.08 \mathrm{Nm}$ at its front knees, the algorithm reduces the lengths of the front leg links to create near singular configurations. On the other hand, the algorithm applies minor changes to the rear leg because their torque profiles are predicted to be lower than the front legs. As a result, the algorithm successfully reduces the maximum torque to $1.42 \mathrm{Nm}$, which is $32 \%$ less than the original value (Fig. 7).

For hardware validation, we 3D-print new links with the optimized lengths and assembled a new quadruped (Fig. 8). After fabrication, we replay the new motion plan that is also concurrently optimized with the design parameters. As the 

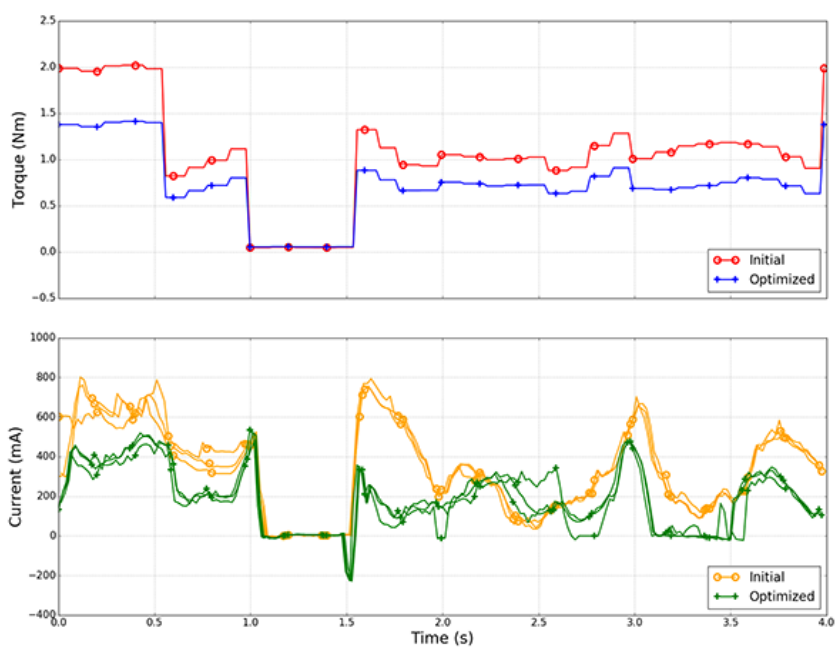

Fig. 7. Comparison of the front right knee torques in simulation (top) and on fabricated hardware (bottom).
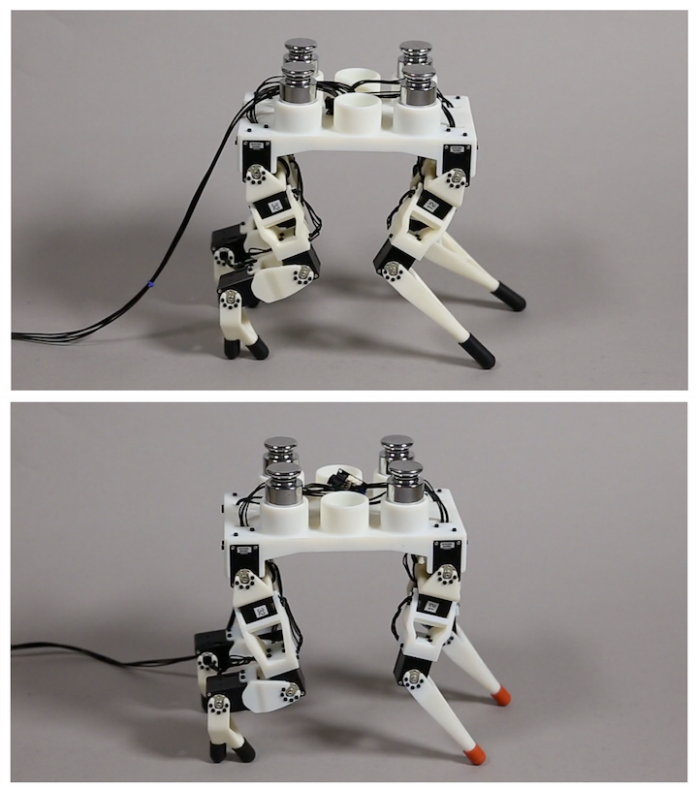

Fig. 8. Comparison of the original (top) and optimized (bottom) small quadrupeds with rotary actuators.

actuators do not have torque sensors, we use the maximum current as the performance criteria. Figure 7 shows the current profiles of two designs at the front right knee for a single cycle of the motion. The maximum current is successfully reduced to $535 \mathrm{~mA}$ in the optimized design, which is $30 \%$ less than the original maximum current $(766 \mathrm{~mA})$ and roughly matches the reduction ratio in simulation. The predicted torque and actual current profiles do not exactly match but show a large positive Pearson's correlation coefficient of 0.7 .

\section{Discussion AND Future Work}

We presented a novel algorithm for optimizing robot design parameters, such as link lengths and actuator layouts, and the associated motion parameters including joint positions, actuator forces, and contact forces at every frame. To guide the optimization, our algorithm first linearizes the local manifold of valid designs implicitly defined by a set of constraints. It then changes the design parameters in the direction of the locally defined gradient. Our problem formulation can also take additional inequality constraints, such as position limits of linear actuators or friction cones of contact forces. We demonstrated that our algorithm is general enough to optimize designs of various types of robots such as manipulators and legged robots, with both linear and rotary actuators. We also validated the optimized designs in simulation as well as on fabricated hardware using off-the-shelf actuators and 3Dprinted links.

There are a few possible directions for future work.

The presented design optimization algorithm selects one taget parameter and specifies its change, and determines the changes in other parameters required to stay on the constraint manifold. We implemented this simple update rule because it only needs to select a single parameter for each time step. Although we demonstrated that the proposed scheme is sufficient in various optimization scenarios, it may require many iterations to reach the desired design or even fall into sub-optimal solutions. Therefore, it would be interesting to incorporate more complex optimization algorithms into our framework for selecting the target parameters and their desired changes.

Our algorithm can only consider continuous parameters, such as link lengths or actuator forces. For future research, we would like to include discrete variables, such as the number of actuators, footfall patterns, or the number of joints actuated by a single actuator (e.g. monoarticular or biarticular), because they are also critical to robot performance. However, current implementation of the framework cannot handle discrete variables because it requires the first and second derivatives of the constraints with respect to the parameters. For instance, the moment arm of a linear actuator discontinuously changes when the actuator switches between monoarticular and biarticular configurations.

In all examples, our input motion plans are quite simple consisting of only a single instance of manipulation or locomotion, resulting in designs specialized for the given motion plan. It would be also interesting to optimize the design for a family of parameterized motion plans, such as walking with different turning angles. We could also consider tasks that require more complex interactions with the environment, such as climbing up stairs.

\section{REFERENCES}

[1] Atlas, http://www.bostondynamics.com/robot_Atlas.html.

[2] HydroLek, http://www.hydro-lek.com/manipulators.php.

[3] Spot, https://youtu.be/M8YjvHYbZ9w, .

[4] SpotMini, https://youtu.be/tf7IEVTDjng, .

[5] Marco Ceccarelli and Chiara Lanni. A multi-objective optimum design of general $3 \mathrm{R}$ manipulators for pre- 
scribed workspace limits. Mechanism and Machine Theory, 39(2):119-132, 2004.

[6] Nick Cheney, Robert MacCurdy, Jeff Clune, and Hod Lipson. Unshackling Evolution: Evolving Soft Robots with Multiple Materials and a Powerful Generative Encoding. Proceeding of the Fifteenth Annual Conference on Genetic and Evolutionary Computation, 2013.

[7] Jean-François Collard, P. Fisette, and P. Duysinx. Contribution to the Optimization of Closed-Loop Multibody Systems: Application to Parallel Manipulators. Multibody System Dynamics, 13(1):69-84, 2005.

[8] Alessandro Crespi, Konstantinos Karakasiliotis, Andre Guignard, and Auke Jan Ijspeert. Salamandra Robotica II: An amphibious robot to study salamander-like swimming and walking gaits. IEEE Transactions on Robotics, 29(2):308-320, 2013.

[9] Dynamixel. http://robotis.com, 2016.

[10] Thomas Geijtenbeek, Michiel van de Panne, and a. Frank van der Stappen. Flexible muscle-based locomotion for bipedal creatures. ACM Transactions on Graphics, 2013.

[11] Knut Graichen, Sebastian Hentzelt, Alexander Hildebrandt, Nadine Kärcher, Nina GaiBert, and Elias Knubben. Control design for a bionic kangaroo. Control Engineering Practice, 42:106-117, 2015.

[12] Sehoon Ha, Stelian Coros, Alexander Alspach, Joohyung Kim, and Katsu Yamane. Task-based Limb Optimization for Legged Robots. International Conference on Intelligent Robots and Systems, 2016.

[13] K Jittorntrum. An implicit function theorem. Journal of Optimization Theory and Applications, 1978.

[14] Eric Jones, Travis Oliphant, and Pearu Peterson. $\{$ SciPy $\}$ : open source scientific tools for $\{$ Python $\} .2014$.

[15] Chang Doo Jung, Won Jee Chung, Jin Su Ahn, Myung Sik Kim, Gi Soo Shin, and Soon Jea Kwon. Optimal mechanism design of in-pipe cleaning robot. IEEE International Conference on Mechatronics and Automation, pages 1327-1332, 2011.

[16] Dongmok Kim, Heeseung Hong, Hwa Soo Kim, and Jongwon Kim. Optimal design and kinetic analysis of a stair-climbing mobile robot with rocker-bogie mechanism. Mechanism and Machine Theory, 50:90-108, 2012.

[17] Sung Gaun Kim and Jeha Ryu. New dimensionally homogeneous jacobian matrix formulation by three endeffector points for optimal design of parallel manipulators. IEEE Transactions on Robotics and Automation, (4), 2003.

[18] Daniel Kuehn, Felix Bernhard, Armin Burchardt, Moritz Schilling, Tobias Stark, Martin Zenzes, and Frank Kirchner. Distributed computation in a quadrupedal robotic system. International Journal of Advanced Robotic Systems, 11(1), 2014. ISSN 17298814. doi: 10.5772/58733.

[19] Chris Leger. Automated Synthesis and Optimization of Robot Configurations : An Evolutionary Approach. Design Engineering, pages 1-234, 1999.

[20] H. Lipson and J.B. Pollack. Towards continuously reconfigurable self-designing robotics. IEEE International Conference on Robotics and Automation. Symposia Proceedings, 2(April):1761-1766, 2000.

[21] Vittorio Megaro, Bernhard Thomaszewski, Maurizio Nitti, Otmar Hilliges, Markus Gross, and Stelian Coros. Interactive Design of 3D-Printable Robotic Creatures. ACM Transactions on Graphics, 2015.

[22] Christiaan J Paredis and Pradeep K Khosla. An Approach for Mapping Kinematic Task Specifications into a Manipulator Design. International Conference on Advanced Robotics, 1, 1991.

[23] PyDART. A Python Binding of Dynamic Animation and Robotics Toolkit, http://pydart2.readthedocs.io.

[24] Xuewen Rong, Yibin Li, Jiuhong Ruan, and Bin Li. Design and simulation for a hydraulic actuated quadruped robot . Journal of mechanical science and technology, 2012. doi: 10.1007/s12206-012-0219-8.

[25] Claudio Semini, Nikos G Tsagarakis, Emanuele Guglielmino, Ferdinando Cannella, and Darwin G Caldwell. Design of HyQ a Hydraulically and Electrically Actuated Quadruped Robot. Proceedings of the Institution of Mechanical Engineers, Part I: Journal of Systems and Control Engineering, 2011.

[26] Sangok Seok, Albert Wang, Meng Yee Chuah, Dong Jin Hyun, Jongwoo Lee, David M. Otten, Jeffrey H. Lang, and Sangbae Kim. Design Principles for Energy-Efficient Legged Locomotion and Implementation on the MIT Cheetah Robot. IEEE/ASME Transactions on Mechatronics, 20(3):1117-1129, 2014.

[27] Karl Sims. Evolving Virtual Creatures. ACM Transactions on Graphics, (July):15-22, 1994.

[28] E.J. Van Henten, D.A. Vant Slot, C.W.J. Hol, and L.G. Van Willigenburg. Optimal manipulator design for a cucumber harvesting robot. Computers and Electronics in Agriculture, 65(2):247-257, 2009.

[29] Kevin Wampler and Zoran Popović. Optimal gait and form for animal locomotion. ACM Transactions on Graphics, 28:1, 2009.

[30] Yuan Yun and Yangmin Li. Optimal design of a 3-PUPU parallel robot with compliant hinges for micromanipulation in a cubic workspace. Robotics and ComputerIntegrated Manufacturing, (6), 2011. 